\title{
La resistencia a los antibióticos: un problema muy serio
}

\section{Resistance to antibacterial agents: A serious problem}

Correspondencia

Ciro Maguiña Vargas

ciro.maguina@upch.pe

\section{Recibido: 15/02/2019}

Arbitrado por pares

Aprobado: 08/05/2019

\section{Citar como: González Mendoza J,} Maguiña Vargas C, González Ponce FM. La resistencia a los antibióticos: un problema muy serio. Acta Med Peru. 2019;36(2):145-51
Jorge González Mendoza1,2,3ab , Ciro Maguiña Vargas¹cde, Flor de María González Ponce ${ }^{1,4 f}$

1 Universidad Peruana Cayetano Heredia. Lima, Perú.

2 London School of Hygiene and Tropical Medicine. London, Reino Unido.

3 Universidad Peruana de Ciencias Aplicadas. Lima, Perú.

4 Hospital Nacional Cayetano Heredia. Lima, Perú.

a Especialista en Enfermedades Infecciosas y Tropicales, b Master of Science, Infection and Health in the Tropics, c Especialista en Enfermedades Infecciosas y Tropicales y Dermatología, d Doctor en Medicina, e Director de los Institutos de Investigación f Médico Residente en la Especialidad de Enfermedades Infecciosas y Tropicales.

\section{RESUMEN}

El uso de los antibióticos desde los años 40 del siglo pasado permitió disminuir en forma importante y notable la morbilidad y mortalidad a nivel mundial. Sin embargo, la aparición de la resistencia antimicrobiana ha hecho que el tratamiento de las enfermedades infecciosas, se vuelva una tarea desafiante para el médico que debe brindar opciones terapéuticas, racionales y basadas en evidencias para mejorar la salud de los pacientes. Esta revisión brinda una visión panorámica sobre la gravedad de este problema y el papel preponderante que deben asumir los sistemas de salud en el apoyo a los profesionales de la salud y en la educación de los pacientes para llegar al ansiado uso racional de estos medicamentos.

Palabras clave: Farmacorresistencia microbiana; Utilización de medicamentos; Uso excesivo de los servicios de salud (fuente: DeCS BIREME).

\section{ABSTRACT}

The use of antibiotics since mid-twentieth century significantly reduced morbidity and mortality worldwide. However, the emergence of antimicrobial resistance has made therapy of infectious diseases a challenging task for physicians, who must provide therapeutic, rational and evidence-based options aiming to improve their patients' health. This review provides an overview on how serious the current situation and the paramount role is that health systems must play in supporting healthcare professionals and educating patients in order to achieve rational use of these medications.

Keywords: Drug resistance, microbial; Drug utilization; Medical overuse (source: NLM MeSH). 
Enfermedades infecciosas: breve presentación de la situación actual

En países de medianos-bajos ingresos; y en nuestro país en particular, si bien se ha encontrado un viraje epidemiológico a una mayor frecuencia de enfermedades no transmisibles, particularmente las condiciones cardio-metabólicas y las neoplasias malignas como principales causas de morbilidad ${ }^{[1,2]}$; las enfermedades infecciosas por sí mismas, o como complicaciones de las condiciones ya mencionadas, siguen siendo causas importantes de morbilidad y mortalidad en estos países ${ }^{[3]}$.

En este contexto, muchas enfermedades infecciosas, tales como tuberculosis, malaria, enfermedad diarreica aguda, infecciones respiratorias agudas y un largo etcétera abarcan una gran parte de la morbilidad y mortalidad causadas por diversos agentes patógenos. A ello se ha sumado la aparición de numerosas y nuevas enfermedades de origen bacteriano, viral y micótico, tales como las infecciones por Legionella pneumophila, Campylobacter, nuevas especies del género Bartonella ( $B$. henselae, B. quintana, B. elizabethae), MRSA (Staphylococcus aureus meticilinorresistente), Hafnia, Edwarsiella, Rotavirus, Calicivirus, Cyclospora cayetanensis, Cryptosporidium parvum, Micobacterias atípicas, Hantavirus, Ébola, Lassa virus, Virus Guanarito, Virus Sabia, Coronavirus (causante del Síndrome respiratorio agudo severo, SRAS), HTLV1, VIH/SIDA, Balamuthia mandrilaris $\mathrm{y}$, últimamente, las diversas variedades de los virus influenza, entre otros ${ }^{[4,5]}$.

Centrándonos en las infecciones bacterianas, propósito principal de la presente revisión, debemos señalar que los antibióticos han sido, son y - si los utilizamos juiciosamente - seguirán siendo importantes armas para el tratamiento de muchas dolencias infecciosas, algunas de las cuales causaban gran mortalidad. Así, el uso de los antibióticos desde los años 40 del siglo pasado permitió disminuir en forma importante y notable la morbilidad y mortalidad de algunos de estos males; y se pensó en forma equivocada que muchas de estas enfermedades iban a desaparecer ${ }^{[6,7]}$.

\section{Orígenes de la resistencia a los antibióticos}

Después de la introducción de los primeros antibióticos, rápidamente comenzaron a aparecer los reportes de resistencia a dichos compuestos por parte de las bacterias, a través de diversos mecanismos ${ }^{[8]}$. Alexander Fleming, ganador del Premio Nobel por el descubrimiento de la penicilina, ya había advertido acerca de los riesgos de la resistencia a los antibióticos; a pesar de ello, a unos 10 años de la introducción a gran escala del citado antibiótico, comenzaron a reportarse los primeros casos de resistencia ${ }^{[9,10]}$.

Este fenómeno ha progresado de tal manera que la multidrogorresistencia de las bacterias a los antibióticos tanto de uso común como a aquellos de uso restringido ha dejado de ser una ocurrencia inusual para convertirse en parte de nuestra práctica diaria, a todo nivel, principalmente en las unidades de cuidados intensivos, pasando por las áreas de hospitalización y emergencia, así como a nivel de la comunidad; y, en algunos casos, en lugares insospechados, habiéndose reportado presencia de genes de multidrogorresistencia a antibióticos en tribus de nativos sudamericanos que nunca habían tenido contacto con lo que denominamos 'civilización' [11]. A lo largo de los años, cada vez más microorganismos, los cuales han sido expuestos a crecientes cifras de agentes antibacterianos en las más variadas concentraciones, eventualmente han desarrollado resistencia a prácticamente todos los antibióticos en uso.

Otra situación que está llamando cada vez más atención es el enorme uso de antibióticos en la ganadería y - en un grado algo menor - en la agricultura. Ello se traduce en enormes cantidades de antibióticos liberadas hacia el medio ambiente, generando una presión selectiva a favor de los microorganismos resistentes. Hollis y Ahmed reportaron que cerca del $80 \%$ de los antibióticos que se consumen en los EE.UU. se usan en la agricultura y la acuicultura. En las granjas, estos compuestos son parte de los programas de engorde y mejoramiento de los animales; también se agregan a la alimentación de los salmones de crianza; y este uso con una mentalidad de corto plazo (la ganancia en los negocios) está contribuyendo con una crisis de gran magnitud ${ }^{[12]}$. Los consumidores ingerimos dosis subterapéuticas de los antibióticos que son suficientes para generar poblaciones menos vulnerables en las bacterias que sobreviven. Estas, a su vez, intercambian genes que confieren resistencia; $y$, entonces se tienen nuevas cepas de bacterias multirresistentes, las cuales, bajo determinadas circunstancias, habrán de causar infecciones serias y más que potencialmente letales ${ }^{[13]}$.

En los últimos años, la aparición de numerosos mecanismos de generación de resistencia a los antibióticos, ha hecho que el manejo de las enfermedades infecciosas se vuelva un asunto complejo, sin el apoyo del desarrollo de nuevos compuestos con actividad antibacteriana (Tabla 1).

El número de nuevos antibióticos aprobados en las últimas décadas ha disminuido marcadamente; y algunos de los nuevos agentes que parecían ser promisorios en cuanto a su actividad antibacteriana han tenido que ser retirados del mercado por diversas reacciones adversas serias, algunas potencialmente letales ${ }^{[14]}$. En consecuencia, se hace imperativo tener un uso juicioso, apropiado; y - de acuerdo con las circunstancias controlado de los antibióticos en las diversas especialidades de la medicina. Debe recordarse que si bien la resistencia a los antibióticos es el tema que requiere el mayor esfuerzo, no debe soslayarse la importancia de las reacciones adversas y la toxicidad relacionada con el uso de estos medicamentos.

\section{Antibióticos en la práctica diaria}

Otro escenario en el cual se debe poner mucho énfasis en referencia al uso racional y/o juicioso de los antibióticos es la consulta externa; y, en consecuencia, en la comunidad. Las infecciones del tracto respiratorio son una de las principales razones para consultar, tanto en pediatría como en medicina de adultos, seguidas por las infecciones gastrointestinales y de la piel, entre otras condiciones adicionales ${ }^{[15,16]}$. 
Tabla 1. Mecanismos de resistencia de acuerdo al tipo de antibiótico.

Tipo de antibiótico (ejemplos)

$\beta$-Lactámicos (Penicilinas, Cefalosporinas, Carbapenems, Monobactámicos)

Aminoglicósidos (Gentamicina, Estreptomicina, Espectinomicina)

Glicopéptidos (Vancomicina, Teicoplanina)

Tetraciclinas (Minociclina, Tigeciclina)

Macrólidos (Eritromicina, Azitromicina)

Lincosamidas (Clindamicina)

Estreptograminas (Synercid)

Oxazolidinonas (Linezolid)

Fenicoles (Cloramfenicol)

Quinolonas (Ciprofloxacina)

Pirimidinas (Trimetoprim)

Sulfonamidas (Sulfametoxazol)

Rifamicinas (Rifampicina)

Lipopéptidos (Daptomicina)

Péptidos catiónicos (Colistina)

\section{Modos de resistencia}

Hidrólisis, eflujo, modificación de receptores

Fosforilación, acetilación, nucleotidilación, eflujo, modificación de receptores

Reprogramación de la biosíntesis del péptidoglucano

Mono oxigenación, eflujo, modificación de receptores

Hidrólisis, glicosilación, fosforilación, eflujo, modificación de receptores

Nucleotidilación, eflujo, modificación de receptores

Liasa C-O (estreptograminas de tipo B), acetilación

(estreptograminas de tipo A), eflujo, modificación de receptores

Eflujo, modificación de receptores

Acetilación, eflujo, modificación de receptores

Acetilación, eflujo, modificación de receptores

Eflujo, modificación de receptores

Eflujo, modificación de receptores

Ribosilación de la ADP, eflujo, modificación de receptores

Modificación de receptores

Modificación de receptores, eflujo

Fuente: Adaptado del trabajo de Davies J, Davies D. Origins and Evolution of Antibiotic Resistance. Microbiol Mol Biol Rev. $2010 ; 74: 417-433$.

Muchas de las prescripciones de antibióticos se realizan en forma empírica, superando en diversos estudios un $50 \%$ de los casos $^{[17,18]}$. Adicionalmente, se debe considerar que cerca de una tercera parte a la mitad de las prescripciones de antibióticos son por infecciones del tracto respiratorio; y siendo la mayor parte de estas infecciones de origen viral, está demostrado que los antibióticos no afectan la duración, los síntomas y la intensidad de la enfermedad. Aquí intervienen diversos factores que pasan tanto por los médicos como por los pacientes; por ejemplo, existe en algunos profesionales de la salud la errónea creencia de 'proteger' al paciente ante la 'posibilidad' de una infección bacteriana posterior, concepto que carece de sustento, pero muy arraigado ${ }^{[19]}$.

Por otro lado, existe la sensación de tranquilidad que otorga a algunas personas la prescripción de un antibiótico. Se confunde la utilidad de tales medicamentos con la búsqueda del alivio sintomático; y ocasionalmente algunos pacientes hasta demandan tal tipo de tratamiento. Por ejemplo, hay escenarios comunes en el uso ambulatorio de los antibióticos, como la automedicación, las enfermedades febriles, los casos de infecciones en consulta externa, la interrupción del tratamiento con antibióticos ante la disminución o mejoramiento de los síntomas ${ }^{[20]}$.

Adicionalmente, a esta situación añadimos algunas situaciones que enfrentamos en la práctica diaria, como las siguientes:
- Desconocimiento y falta de confianza: cuando algunos médicos no están seguros del diagnóstico en un paciente febril (acompañado de síntomas respiratorios o gastrointestinales), deciden prescribir algún antibiótico para 'no perder la confianza del paciente'. Este último percibe que recibe un tratamiento; y en algunos casos por consideraciones o presiones de índole legal (temor a que un supuesto mal manejo sea considerado como negligencia).

- Presión por parte del paciente (y/o sus familiares): muchas personas tienen acceso a diversas fuentes de información; $y$ en ocasiones exigen ser tratados con aquel producto sobre el cual han leído. Aquí hay que ser cuidadoso, cordial y tajante a la vez. El responsable del acto médico es el profesional de la salud, quien tiene el conocimiento y el soporte legal para emitir una prescripción; y no porque tal medicamento sea de preferencia del paciente.

- Condiciones del mercado: aunque suene trivial, en muchas ocasiones algunos médicos prefieren hacer una prescripción de un antibiótico, llevados por su inseguridad y desconocimiento, puesto que no desean perder al 'cliente' (en este caso el paciente).

Tomando como ejemplo algunas de las condiciones que encontramos con mayor frecuencia en la consulta externa: infecciones del tracto respiratorio, del tracto urinario, infecciones de la piel y de los tejidos blandos, tenemos algunas observaciones 
no solamente basadas en evidencia, sino en el sentido común, como, por ejemplo:

- Saber tratar los síntomas (o postergar el inicio de un antibiótico) ante la sospecha de un origen viral en la condición (infecciones del tracto respiratorio).

- Verificar cuidadosamente las características clínicas (secreción francamente purulenta vs. costra mielicérica) en el caso de las infecciones de la piel y los tejidos blandos.

- Formular preguntas precisas acerca de las molestias del paciente (¿la diarrea es con moco y/o sangre?)

- La identificación del patógeno y la determinación de su sensibilidad permitirá elegir la mejor terapia con la mayor precisión antes que un antibiótico de muy amplio espectro.

- Tener muy en cuenta los factores del huésped. La presencia de enfermedades crónicas subyacentes (por ejemplo, diabetes) y la edad pueden hacerlo más vulnerable a determinadas patologías de origen infeccioso.

- Y, por cierto, en caso de duda, consultar con el especialista en enfermedades infecciosas.

Un último tema-y no por ello menos importante-corresponde a los conocimientos y actitudes de las personas en referencia al (mal) uso de los antibióticos. Se ha reportado que el incumplimiento con los regímenes prescritos de antibióticos y el uso inapropiado de éstos están fuertemente asociados con el conocimiento sobre estos medicamentos; el cual, a su vez, es influenciado por diversos factores, destacando el nivel educacional, el ingreso familiar y la falta de orientación por parte de un profesional de la salud ${ }^{[21]}$.

Un estudio realizado en una comunidad en nuestro medio demostró que dos terceras partes de los pacientes que acudieron a la consulta externa recibieron prescripciones de antibióticos; con grados variables de conocimiento acerca de dichos medicamentos en la población y bajos porcentajes de automedicación ${ }^{[22]}$. Sin embargo, los encuestados (tanto los pacientes como el personal médico) coincidieron en que el uso inadecuado de los antibióticos es un problema de salud pública a nivel mundial, concordantemente con lo señalado en la estrategia mundial para la contención de la resistencia a lo antimicrobianos propuesta por la Organización Mundial de la Salud, en donde se señala que es necesario mejorar el uso de estos medicamentos mediante la supervisión y divulgación de guías de práctica clínica ${ }^{[23]}$. Adicionalmente, más de dos tercios de los encuestados consideraron que había una sobreutilización de los antibióticos en ambos centros de salud y a nivel nacional. Un punto a destacar en la citada referencia es que cerca de una tercera parte de los profesionales de la salud encuestados tuvieron errores en el manejo de los antibióticos ante casos hipotéticos que les fueron presentados ${ }^{[22]}$.

Otro aspecto importante dentro de este tema es la automedicación, definida como comprar libremente un antibiótico, sin una prescripción médica; y administrarse el producto (o a otros integrantes de la familia, particularmente a los niños) queriendo tratar una supuesta condición infecciosa a partir de una percepción errónea de la misma ${ }^{[24,25]}$; y las tasas de automedicación son muy variables en diversos países, fluctuando entre $5 \%$ a $45 \%$, incluyendo estudios en el mundo desarrollado ${ }^{[26,27]}$.

\section{Escenarios en la resistencia a los antibióticos y propuestas para enfrentar el problema}

Numerosos estudios sobre el diagnóstico y la vigilancia de la resistencia a los antibióticos han sido realizados en diversas partes del mundo, muchos de los cuales han producido resultados muy interesantes. Cabe señalar que no se puede - ni se debe - extrapolar los hallazgos de las diferentes áreas geográficas, ni mucho menos de los diferentes servicios en un hospital, puesto que la flora bacteriana causante de las diversas patologías infecciosas puede ser marcadamente variable y variada ${ }^{[28,29]}$.

Puesto así el escenario, se han formulado recomendaciones acerca del uso racional de los antibióticos en los diversos escenarios del trabajo en salud, sea el medio hospitalario, las unidades de cuidados intensivos y la consulta externa, enfatizándose la necesidad de una vigilancia continua y permanente, puesto que la capacidad de mutación de las bacterias y su continua adaptación al medio ambiente les hace adquirir características de resistencia, de manera tal que en la mayoría de las veces están no solo uno sino varios pasos por delante de nosotros ${ }^{[30]}$.

La idea de tener programas de seguimiento y vigilancia del uso de antibióticos en los establecimientos de salud tiene, entre otros objetivos, la finalidad de optimizar la evolución clínica de los pacientes en el manejo de las diversas patologías infecciosas que los aquejan, estableciendo los patrones de microbiología, resistencia y sensibilidad a los antibióticos en el medio, las características del uso de tales compuestos en las facilidades de salud; generar guías para el tratamiento de las diversas patologías infecciosas y hacer que tales guías se cumplan, para que así se minimice la resistencia y, en consecuencia, los costos de atención.

Existen ocasiones en que se dan limitaciones al uso de ciertos antibióticos por consideraciones de costos de tratamientos de pacientes individuales; pero tiene que quedar claro que cuesta mucho más para cualquier sistema de salud tener un paciente aquejado por una infección causada por un microorganismo multirresistente; más aun si este es de origen nosocomial. Al respecto de ello, el uso de los antibióticos, particularmente aquellos de amplio espectro y máxima potencia (los denominados de 'tercera' o 'cuarta' generación, independientemente de las clases a las cuales pertenezcan, debiera estar firmemente regulado, para indicaciones precisas y tiempos establecidos de acuerdo con los protocolos.

Cierto es que tales guías de tratamiento tienen un rol orientador, su nombre lo dice, 'guías', siendo finalmente la 
evidencia bacteriológica, la condición clínica del paciente; y, por cierto, el buen juicio, el conocimiento y la experiencia del médico tratante, sobre la base de la evidencia disponible en la literatura, los que determinarán el agente antibacteriano a utilizar en aquel paciente con septicemia, neumonía nosocomial, sepsis post-quirúrgica, endometritis, infección complicada del tracto urinario o peritonitis generalizada, por citar algunos ejemplos ${ }^{[31]}$.

Los programas de supervisión y control del uso de los antibióticos deben ser sostenibles en el tiempo y no ser esfuerzos aislados ${ }^{[32]}$. Hemos sido testigos en más de una ocasión de cómo con mucho entusiasmo se iniciaron tales programas, para que posteriormente se hayan diluido en el tiempo, por una serie de razones. La tarea no es solamente de los especialistas en enfermedades infecciosas; ello corre a cargo de un equipo multidisciplinario en donde deben estar firmemente comprometidos no solamente el personal médico, sino también el personal de enfermería, el personal técnico y auxiliar, farmacia, mantenimiento y la parte administrativa. Hacemos énfasis en este último componente porque no siempre tenemos la mejor llegada o comunicación con esta área, y debemos recordar que son ellos quienes en muchas ocasiones tienen la palabra final en la toma de decisiones referentes a compras de insumos, medicamentos y diversos materiales.

En esta época de búsqueda constante de eficiencia y efectividad, de ahorros de costos y limitar gastos, tales esfuerzos siempre deben estar orientados a garantizar la máxima seguridad para los pacientes. Algunas veces se tiene una visión de corto plazo que solamente se enfoca en el momento de determinada infección y la prescripción del agente antibacteriano correspondiente, sin mirar las consecuencias en el mediano y en el largo plazo. La vigencia de un programa para el control del uso racional de los antibióticos en los establecimientos de salud a la larga reduce los costos, puesto que se evitan las infecciones multirresistentes con las complicaciones que ya se han mencionado; y existen reportes que señalan elevaciones en los costos con la discontinuación de tales programas ${ }^{[33]}$. Los análisis de costo-beneficio tan en boga en la actualidad no solamente han de restringirse a los ahorros potenciales en el uso de medicamentos, sino a la menor estancia en el hospital, las menores duraciones de los tratamientos, la mayor y mejor seguridad para los pacientes; $y$, definitivamente, las menores tasas de mortalidad, aunque bien sabemos que no existe análisis que pueda estimar el valor de una vida humana perdida ${ }^{[34]}$.

A nivel general podemos entonces tomar los siguientes puntos de reflexión, sobre la base de lo consensuado en las conclusiones de la Reunión de Uppsala sobre la Salud (Uppsala Health Summit) celebrada en Junio del $2015^{[35]}$.

- Los gobiernos nacionales y las organizaciones internacionales, tales como la Unión Europea o las Naciones Unidas, deberán comprometerse más en la lucha contra la resistencia a los antibióticos. El compromiso de los gobiernos y su capacidad de liderazgo con cruciales en este aspecto.
- Se necesita diseminar la información y facilitar la educación de los profesionales de la salud, el público consumidor, los granjeros y otros involucrados en el tema. Los gobiernos y los organismos especializados, tales como la Organización Mundial de la Salud, habrán de coordinar y asegurar la calidad de la información a nivel nacional o internacional. Los mensajes deberán ser especialmente adaptados a quienes están dirigidos. Una comunicación responsable puede ayudar a mantener el tema con prioridad en la agenda política, así como en las mentes de los involucrados.

- Las políticas de restricción de los antibióticos no deberán afectar los ingresos de las partes involucradas. Deberán perfeccionarse los modelos de negocios, de manera tal que no se generen mayores perjuicios económicos. Así, las regulaciones comerciales, los acuerdos de negocios y las políticas públicas en su conjunto serán herramientas para promover el uso racional de los antibióticos.

- El uso racional de los antibióticos implica tener acceso a medicamentos efectivos y a una buena capacidad de los profesionales de la salud, herramientas para el diagnóstico adecuadas e información actualizada y constante acerca de la resistencia a los antibióticos. Deberá reprimirse la venta de antibióticos sin receta; y explorar y combatir los incentivos perversos para el sobreuso de tales compuestos.

- El desarrollo de nuevos tratamientos, vacunas y herramientas para el diagnóstico depende no solo de los recursos financieros, sino que constituye un reto enrome desde el punto de vista científico. Es imprescindible el esfuerzo conjunto de la academia, la industria y el estado, evitando la duplicidad de esfuerzos y compartiendo la información. La prioridad es, por cierto, atender las necesidades más urgentes.

- El costo de no actuar supera los cálculos más pesimistas. Algunos autores señalan que, con la actual progresión de la multirresistencia a los antibióticos, en relativamente pocas décadas podríamos retornar a la era pre-antibiótica.

\section{¿Entonces, cómo y cuándo recetar un antibiótico?}

Puede superarse el surgimiento de las bacterias resistentes a los antibióticos promoviendo el uso óptimo de los antibióticos disponibles y evitar la transmisión de los microorganismos multirresistentes a través de las diversas medidas para el control de las infecciones ${ }^{[31]}$. Las siguientes proposiciones pueden ayudar a los clínicos a asegurar el uso apropiado de los antibióticos ${ }^{[36]}$.

- Un buen diagnóstico clínico, sobre la base de una historia clínica centrada tanto en la enfermedad actual como en los antecedentes, considerando las posibles exposiciones, viajes, medicamentos de uso habitual, contacto con animales, hábitos personales y diversos aspectos adicionales.

- Determinar si realmente es necesario el antibiótico, sobre la base de ponderar los riesgos (resistencia, efectos adversos) contra los beneficios (eficacia, recuperación rápida) y el 
costo. Reconocer qué procesos infecciosos son autolimitados y solo requieren sintomáticos.

- Reservar la terapia antibiótica empírica para aquellas situaciones en donde es imperativo iniciar un antibiótico. No podemos esperar los resultados de los cultivos si se sospecha una neumonía, una infección del tracto urinario alto o sepsis, por citar algunos ejemplos. Ciertamente, dicha terapia será orientada no solamente por la condición clínica del paciente, sino por los microorganismos prevalentes en la comunidad o en el contexto en donde se haya adquirido la infección. Se iniciará con antibióticos de amplio espectro, para luego usar el agente específico contra el patógeno causante, una vez que los cultivos lo hayan identificado.

- Existen situaciones en donde es necesario utilizar combinaciones de antibióticos, los cuales deberán tener mecanismos de acción diferentes y una acción sinérgica. Ello también está relacionado con lo señalado en el párrafo anterior, específicamente el caso de los pacientes en una condición crítica por sepsis.

- Tener en cuenta no solamente el espectro y la acción de determinado antibiótico, sino su capacidad de penetración en el área del organismo en donde se desea que actúe. Ello es de particular importancia en las infecciones del sistema nervioso central, de las articulaciones y los huesos, así como en los casos de prostatitis.

- Deberemos considerar tanto la farmacocinética como la farmacodinámica al establecer las dosis del antibiótico. Estas deberán ser lo suficientemente elevadas para asegurar la eficacia y lo suficientemente bajas para minimizar el riesgo de toxicidad.

- La severidad de la infección, al igual que conocer la absorción y la biodisponibilidad de los antibióticos, permitirán elegir la mejor ruta de administración. En el caso de las infecciones severas, la nueva información microbiológica, la mejora en los títulos de algunos indicadores; y, por cierto, la mejoría clínica del paciente, determinarán el paso precoz a la vía oral.

- Existen factores de los pacientes a ser considerados al prescribir un antibiótico, comenzando con la edad, puesto que los extremos de la vida son más vulnerables a determinadas infecciones y tienen características particulares en cuanto a la absorción, distribución, metabolismo y eliminación de los antibióticos.

- Otros factores a tomarse en cuenta son la función renal y hepática; y en el primer caso, hay que ser particularmente cuidadoso con aquellos medicamentos cuyas dosis deben ser reguladas en los casos de disfunción renal, al igual que con aquellos que por su naturaleza son nefrotóxicos, tal como es el caso de los aminoglicósidos.

- Ciertamente, el embarazo y la lactancia son periodos importantes y críticos. Existen antibióticos cuyo uso está prohibido durante la gestación (por ejemplo, las tetraciclinas); y otros compuestos en los cuales hay que ponderar el riesgo potencial contra el beneficio a obtener.

- Las alergias o la intolerancia a ciertos antibióticos son datos que no debemos obviar al tomar la historia clínica.

- El uso reciente de antibióticos puede orientarnos en la elección del tratamiento en un nuevo cuadro agudo, por la posibilidad de una presión selectiva previa.

Por cierto, la clave del éxito está en una buena comunicación y una cordial relación con el paciente y con sus familiares inmediatos, en darle las indicaciones en forma clara; recordarles que los medicamentos son para su uso personal y que no deben compartirse; y que, si quedara algún remanente del tratamiento, este deberá descartarse apropiadamente.

\section{CONCLUSIONES}

En conclusión, arribamos a lo señalado por diversos autores en múltiples referencias; y que ha sido sintetizado por la Organización Mundial de la Salud en un importante documento emitido hace algunos años, pero que no pierde vigencia: "El uso racional de los medicamentos (en este caso los antibióticos) requiere que los pacientes reciban medicamentos que sean apropiados para sus necesidades clínicas, en las dosis apropiadas para sus requerimientos individuales; durante periodos adecuados de tiempo y con el menor costo tanto para ellos como para sus comunidades". Los antibióticos, anteriormente considerados como una suerte de 'balas mágicas' han representado y representan un enorme avance en la medicina. Su descubrimiento determinó una nueva era en el tratamiento de las enfermedades infecciosas y en la calidad de vida ${ }^{[37-39]}$.

Sin embargo, el uso irracional de los antibióticos, en sus diversas facetas, ha dado como resultado el surgimiento de las bacterias multirresistentes - las denominadas 'superbacterias' según algunos autores - ; y las infecciones causadas por tales microorganismos requieren de acción urgente, puesto que tienden a tener una mayor duración, pueden aumentar el riesgo de complicaciones y aun llevar a la muerte a los pacientes. En consecuencia, es esencial el uso óptimo de los antibióticos, a fin de evitar este creciente problema. La mejor - y tal vez la única - forma de evitar la diseminación de este fenómeno de la multidrogorresistencia es el uso racional de los antibióticos. Su empleo deberá hacerse solo cuando sea estrictamente necesario ${ }^{[36]}$.

Contribución de los autores: Todos los autores contribuyeron en la concepción, diseño, escritura. revisión critica y aprobación del manuscrito final a ser publicado.

Financiamiento: Esta revisión ha sido autofinanciada.

Conflictos de interés: Los autores declaran no tener ningún conflicto de interés. 


\section{REFERENCIAS BIBLIOGRÁFICAS}

1. Organización Panamericana de la Salud (Perú). Cada año, las enfermedades no transmisibles provocan 16 millones de defunciones prematuras, por lo que la OMS insta a redoblar esfuerzos [Internet]. Lima: OPS/OMS; 2015 [citado el 14 de octubre de 2018]. Disponible en: https://www.paho.org/per.../ index. php?option=com content\&view $=$ article $\& i d=2840$ : cadaano-las-enfermedades-no-transmisibles-provocan-16-millonesde-defunciones-prematuras-por-lo-que-la-oms-insta-a-redoblaresfuerzos\&|temid=900

2. Venegas Ojeda D. Situación de las enfermedades crónicas no transmisibles: problema cáncer [Internet]. Lima: MINSA; 2012 [citado el 14 de octubre de 2018]. Disponible en: http://bvs.minsa. gob.pe/local/minsa/2283.pdf

3. Imtiaz F. Infectious diseases burden and surveillance: an epidemiological survey. Epidemiol. 2014;4(2):1000e116.

4. Mukherjee S. Emerging infectious diseases: epidemiological perspective. Indian J Dermatol. 2017;62(5):459-67.

5. National Institutes of Health (US), Biological Sciences Curriculum Study. Understanding Emerging and Re-emerging Infectious Diseases [Internet]. Bethesda, MD: National Institutes of Health (US); 2007 [citado el 14 de octubre de 2018]. Disponible en: https:// www.ncbi.nlm.nih.gov/books/NBK20370/

6. Gannon F. The end of optimism? EMBO Rep. 2008;9(2):111.

7. Zaman SB, Hussain MA, Nye R, Mehta V, Mamun KT, Hossain N. A review on antibiotic resistance: alarm bells are ringing. Cureus. 2017;9(6):e1403.

8. Fariña N. Bacterial resistance. A global public health problem with difficult solution. Mem Inst Investig Cienc Salud. 2016;14(1):6-7.

9. Bushak L. A brief history of antibiotic resistance: how a medical miracle turned into the biggest public health danger of our time [Internet]. New York: Medical Daily LLC; 2016 [citado el 14 de octubre de 2018]. Disponible en: https://www.medicaldaily.com/ antibiotic-resistance-history-373773

10. Rosenblatt-Farrell N. The Landscape of Antibiotic Resistance. Environ Health Perspect. 2009;117(6):A244-50.

11. Clemente JC, Pehrsson EC, Blaser MJ, Sandhu K, Gao Z, Wang $B$, et al. The microbiome of uncontacted Amerindians. Sci Adv. 2015;1(3):e1500183.

12. Hollis $A$, Ahmed Z. Preserving antibiotics, rationally. N Engl J Med. 2013;369(26):2474-6

13. Chang Q, Wang W, Regev-Yochay G, Lipsitch M, Hanage WP. Antibiotics in agriculture and the risk to human health: how worried should we be? Evol Appl. 2015;8(3):240-7.

14. Department of Health and Human Services and Food and Drug Administration. Determination That TEQUIN (Gatifloxacin) Was Withdrawn From Sale for Reasons of Safety or Effectiveness. Federal Register. 2008;73(175):52357-8.

15. Tangcharoensathien V, Chanvatik S, Sommanustweechai A. Complex determinants of inappropriate use of antibiotics. Bull World Health Organ. 2018;96(2):141-4.

16. Al-Homaidan HT, Barrimah IE. Physicians' knowledge, expectations, and practice regarding antibiotic use in primary health care. Int J Health Sci (Qassim). 2018;12(3):18-24.

17. Chem ED, Anong DN, Akoachere J-FKT. Prescribing patterns and associated factors of antibiotic prescription in primary health care facilities of Kumbo East and Kumbo West Health Districts, North West Cameroon. PLoS One. 2018;13(3):e0193353.

18. Adisa R, Orherhe OM, Fakeye TO. Evaluation of antibiotic prescriptions and use in under-five children in Ibadan, SouthWestern Nigeria. Afr Health Sci. 2018;18(4):1189-201.

19. Abera B, Kibret M, Mulu W. Knowledge and beliefs on antimicrobial resistance among physicians and nurses in hospitals in Amhara Region, Ethiopia. BMC Pharmacol Toxicol. 2014;15:26.
20. Kourkouta L, Kotsiftopoulos $\mathrm{CH}$, Papageorgiou M, Iliadis $\mathrm{CH}$, Monios A. The rational use of antibiotics medicine. J Healthc Commun. 2017;2:36.

21. McNulty CAM, Boyle P, Nichols T, Clappison P, Davey P. Don't wear me out--the public's knowledge of and attitudes to antibiotic use. J Antimicrob Chemother. 2007;59(4):727-38

22. González-Ponce F de M. Conocimientos, actitudes y prácticas sobre el uso de antibióticos y la resistencia antimicrobiana en pacientes y médicos de centros de salud de atención primaria de Lima norte, 2014-2015 [Tesis Bachiller]. Lima, Perú: Universidad Peruana Cayetano Heredia; 2017.

23. World Health Organization. The pursuit of responsible use of medicines: sharing and learning from country experiences. Geneva: WHO; 2012.

24. Awad Al, Aboud EA. Knowledge, attitude and practice towards antibiotic use among the public in Kuwait. PLoS One. 2015;10(2):e0117910

25. Awad A, Eltayeb I, Matowe L, Thalib L. Self-medication with antibiotics and antimalarials in the community of Khartoum State, Sudan. J Pharm Pharm Sci. 2005;8(2):326-31.

26. Väänänen $\mathrm{MH}$, Pietilä K, Airaksinen M. Self-medication with antibiotics--does it really happen in Europe? Health Policy. 2006;77(2):166-71.

27. Pfaffenbach G, Tourinho FSV, Bucaretchi F. Self-medication among children and adolescents. Curr Drug Saf. 2010;5(4):324-8.

28. Burnham C-AD, Leeds J, Nordmann P, O'Grady J, Patel J. Diagnosing antimicrobial resistance. Nat Rev Microbiol. 2017;15(11):697-703.

29. Aslam B, Wang W, Arshad MI, Khurshid M, Muzammil S, Rasool MH, et al. Antibiotic resistance: a rundown of a global crisis. Infect Drug Resist. 2018;11:1645-58.

30. Merrett GLB, Bloom G, Wilkinson A, MacGregor H. Towards the just and sustainable use of antibiotics. J Pharm Policy Pract. 2016;9:31.

31. Nathwani D, Sneddon J. Practical guide to antimicrobial stewardship in hospitals [Internet]. France: bioMérieux S.A.; 2013 [citado el 14 de octubre de 2018]. Disponible en: http://bsac.org.uk/wpcontent/uploads/2013/07/Stewardship-Booklet-Practical-Guideto-Antimicrobial-Stewardship-in-Hospitals.pdf

32. Davies J, Davies D. Origins and Evolution of Antibiotic resistance. Microbiol Mol Biol Rev. 2010;74(3):417-33

33. Standiford HC, Chan S, Tripoli M, Weekes E, Forrest GN. Antimicrobial stewardship at a large tertiary care academic medical center: cos analysis before, during, and after a 7-year program. Infect Control Hosp Epidemiol. 2012;33(4):338-45

34. Kaki R, Elligsen M, Walker S, Simor A, Palmay L, Daneman N. Impact of antimicrobial stewardship in critical care: a systematic review. J Antimicrob Chemother. 2011;66(6):1223-30.

35. Uppsala Health Summit 2015: A World Without Antibiotics [Internet]. Uppsala University; 2015 [citado el 14 de octubre de 2018]. Disponible en: https://www.uppsalahealthsummit.se/oursummits/a-world-without-antibiotics-2015/

36. Tiwaskar M, Manohar T. Chapter 2: Rational for Antibiotics Guidelines [Internet]. Mumbai: The Association of Physicians of India; 2017 [citado el 14 de octubre de 2018]. Disponible en: http:// www.apiindia.org/pdf/medicine_update_2017/mu_002.pdf

37. Cascioferro S, Schillaci D. The future of antibiotic: from the magic bullet to the smart bullet. J Microb Biochem Technol. 2014;6(5):1000e118

38. Gastelo RA, Maguiña C. Mecanismos de resistencia bacteriana. Diagnóstico. 2018; 57(2): 82-86.

39. García CA. Resistencia antimicrobiana. En Maguiña C. Uso racional de antibióticos. 4ta edición. Lima: Fondo Editorial del Colegio Médico del Perú; 2018. p 177 -184. 